Check for updates

Cite this: Nanoscale Adv., 2019, 1, 1893

\title{
Top-down fabrication of ordered arrays of GaN nanowires by selective area sublimation $\uparrow$
}

\author{
Sergio Fernández-Garrido, (D) *ab Thomas Auzelle, (D) a Jonas Lähnemann, (D) ${ }^{a}$ \\ Kilian Wimmer, ${ }^{a}$ Abbes Tahraoui ${ }^{a}$ and Oliver Brandt iD a
}

We demonstrate the top-down fabrication of ordered arrays of GaN nanowires by selective area sublimation of pre-patterned $\mathrm{GaN}(0001)$ layers grown by hydride vapor phase epitaxy on $\mathrm{Al}_{2} \mathrm{O}_{3}$. Arrays with nanowire diameters and spacings ranging from 50 to $90 \mathrm{~nm}$ and 0.1 to $0.7 \mu \mathrm{m}$, respectively, are simultaneously produced under identical conditions. The sublimation process, carried out under high vacuum conditions, is analyzed in situ by reflection high-energy electron diffraction and line-of-sight quadrupole mass spectrometry. During the sublimation process, the GaN(0001) surface vanishes, giving way to the formation of semi-polar $\{1 \overline{1} 03\}$ facets which decompose congruently following an Arrhenius temperature dependence with an activation energy of (3.54 \pm 0.07$) \mathrm{eV}$ and an exponential prefactor of $1.58 \times 10^{31}$ atoms per $\mathrm{cm}^{2}$ per $\mathrm{s}$. The analysis of the samples by low-temperature cathodoluminescence spectroscopy reveals that, in contrast to dry etching, the sublimation process does not introduce nonradiative recombination centers at the nanowire sidewalls. This technique is suitable for the topdown fabrication of a variety of ordered nanostructures, and could possibly be extended to other material systems with similar crystallographic properties such as ZnO.

Received 29th November 2018

Accepted 11th March 2019

DOI: $10.1039 / c 8 n a 00369 f$

rsc.li/nanoscale-advances devices with a core-shell geometry. ${ }^{3-8}$ However, despite all these advantages and worldwide research activities, the performance of NW based light-emitting diodes (LEDs) lags behind that of their state-of-the-art two-dimensional counterparts fabricated on $\mathrm{Al}_{2} \mathrm{O}_{3}$ and Si substrates, which nowadays exhibit wall-plug and external quantum efficiencies above $80 \%$ in the blue spectral range. ${ }^{9,10}$ The inferior performance of NW-based LEDs is mainly caused by the limitations and complexities inherent to their formation using bottom-up methods. Particularly, the nonideal growth conditions often required to promote either uniaxial or radial growth may favor the incorporation of higher concentrations of impurities and defects. ${ }^{3,11}$ Furthermore, the different chemical and physical properties of the precursors, which are simultaneously deposited on different crystal facets, can result in the formation of detrimental compositional and structural inhomogeneities. ${ }^{11-13}$

Given that the epitaxial growth of group-III nitrides has reached a high level of maturity, it is appealing to combine this well-developed technology with some of the advantages offered by the NW architecture using a top-down approach. ${ }^{7,14-17}$ It is important to note that top-down methods are also suitable to obtain arrays of largely dislocation free NWs despite the presence of a high density of TDs in the layer used for the top-down process. In fact, the average number of TDs per NW is determined by the product of the TD density of the initial GaN layer and the cross-sectional area of one $\mathrm{NW} .{ }^{15}$ For instance, for a substrate with a TD density on the order of $10^{8} \mathrm{~cm}^{-2}$, typical for $\mathrm{GaN}$ layers grown on $\mathrm{Al}_{2} \mathrm{O}_{3}$, and a NW diameter of $100 \mathrm{~nm}$, less than $1 \%$ of these objects will contain TDs. ${ }^{18}$
${ }^{a}$ Paul-Drude-Institut für Festkörperelektronik, Leibniz-Institut im Forschungsverbund Berlin e.V., Hausvogteiplatz 5-7, 10117 Berlin, Germany. E-mail: sergio. fernandezg@uam.es

${ }^{b}$ Grupo de Electrónica y Semiconductores, Dpto. Física Aplicada, Universidad Autónoma de Madrid, C/ Francisco Tomás y Valiente 7, 28049 Madrid, Spain $\dagger$ Electronic supplementary information (ESI) available: Scanning electron micrographs of the patterned substrate and analysis, by reflection high-energy electron diffraction, of GaN facets formed during thermal sublimation. See DOI: 10.1039/c8na00369f 
The top-down fabrication of NW arrays typically relies on dry etching of a lithographically patterned two-dimensional layer. This fabrication method has, however, not achieved much popularity within the NW community because, in addition to the difficulties in achieving vertical NW sidewalls, the dry etching process inevitably creates point defects acting as nonradiative recombination centers at the NW sidewalls. Significant progress has been made in recent years by introducing an additional anisotropic wet chemical etching step after the creation of NWs by inductively coupled plasma reactive ion etching (ICP-RIE). This additional step removes the damaged material and facilitates the formation of smooth and straight NW sidewalls. ${ }^{15,16}$ Using this method, the fabrication of NW LEDs $^{15}$ and optically pumped NW lasers has been demonstrated. ${ }^{\mathbf{1 6}}$ As an alternative to this two-step etching approach, a new top-down fabrication method coined as selective area sublimation (SAS) was recently demonstrated by Damilano et al. ${ }^{17,19}$ This approach avoids any damage to the NW sidewalls as it is based not on chemical etching but on material sublimation in vacuum, a process used before by different groups to decrease the diameter of as-grown NWs. ${ }^{20-22}$ Damilano et al. utilized this simple method to obtain random arrays of GaN NWs and (In,Ga)N/GaN NW heterostructures. ${ }^{17,19}$

For device applications, it is almost mandatory that the NWs do not have a random arrangement, but form an ordered array with precisely tunable spatial arrangements, NW diameters, and NW-to-NW spacings. In the present work, we explore the fabrication of ordered arrays of Ga-polar ${ }^{23}$ GaN NWs by SAS. We demonstrate that this method is suitable to simultaneously fabricate ordered arrays of NWs with various diameters and spacings on a single wafer. The sublimation process, carried out under ultra high-vacuum conditions, is further analyzed in situ by reflection high-energy electron diffraction (RHEED) and lineof-sight quadrupole mass spectrometry (QMS). A detailed analysis of the decomposition process allows us to assess the temperature dependence of the thermal etching rate, a crucial factor to control the formation by SAS of NWs as well as other types of nanostructures. Finally, we investigate the excitonic transitions from an ordered array of GaN NWs by lowtemperature cathodoluminescence spectroscopy (CL). The emission from the array is found to be more intense than that from the original layer, confirming that the sublimation process does not introduce any nonradiative defects at the NW sidewalls, and revealing an enhanced extraction efficiency of the NW array.

\section{Experimental}

As substrates for SAS, we use commercially available $2^{\prime \prime}$ $\mathrm{GaN}(0001)$ layers grown by hydride vapor phase epitaxy on $\mathrm{Al}_{2} \mathrm{O}_{3}$ (purchased from Suzhou Nanowin Science and Technology). The thickness of the GaN layer is $(4.5 \pm 0.5) \mu \mathrm{m}$ and its TD density $2 \times 10^{8} \mathrm{~cm}^{-2}$. The backside of the substrates is coated with a $1.3 \mu \mathrm{m}$ thick Ti layer for efficient thermal coupling during the sublimation process. To produce the mask for SAS, a $20 \mathrm{~nm}$ thick $\mathrm{Si}_{x} \mathrm{~N}_{y}$ layer is first sputtered on the substrate surface. Afterwards, the $\mathrm{Si}_{x} \mathrm{~N}_{y}$ layer is spin-coated with a $100 \mathrm{~nm}$ thick positive resist. Subsequently, the pattern is written by electron beam lithography (EBL) in a RAITH system. The pattern comprises $100 \times 100 \mu \mathrm{m}^{2}$ fields containing hexagonal arrays of circles. Upon writing the pattern, the resist is developed before depositing $25 \mathrm{~nm}$ of Ni. After a lift-off step, the substrates are diced in $10 \times 10 \mathrm{~mm}^{2}$ pieces and the $\mathrm{Si}_{x} \mathrm{~N}_{y}$ layer is etched at the regions not protected by $\mathrm{Ni}$ using RIE with a $\mathrm{CF}_{4} / \mathrm{O}_{2}$ gas mixture. Finally, the pieces are further etched by ICP-RIE using a mixture of $\mathrm{Ar}, \mathrm{Cl}_{2}$ and $\mathrm{BCl}_{3}$ gases. This final etching step is used to eliminate residual surface contaminants created after etching $\mathrm{Si}_{x} \mathrm{~N}_{y}$ as well as upon the chemical removal of the remaining $\mathrm{Ni}$ layer.

The sublimation of the GaN layer is carried out under ultra high-vacuum conditions in a molecular beam epitaxy system where the process can be monitored in situ by RHEED and QMS. The substrate temperature is measured with an emissivity corrected infrared optical pyrometer from Laytec. Customized Python routines are used to extract the angular intensity profiles around selected GaN diffraction spots of RHEED patterns. Because GaN decomposes congruently in vacuum, ${ }^{24}$ we employ QMS to directly assess the decomposition rate by measuring the desorbing Ga flux. The QMS system response to the $\mathrm{Ga}^{69}$ signal is calibrated in units of atoms per $\mathrm{cm}^{2}$ per $\mathrm{s}$ using the procedure described in ref. 25. Regardless of the surface morphology, we assume that $\mathrm{Ga}$ atoms desorb isotropically.

After the sublimation process, the samples are investigated by scanning electron microscopy (SEM) and low-temperature (14 K) CL spectroscopy and imaging. Scanning electron micrographs are acquired using either a Hitachi S-4800 or a Zeiss Ultra55 field-emission microscope. CL measurements are performed at acceleration voltages of $5 \mathrm{kV}$ using a Gatan MonoCL4 system mounted to the Zeiss Ultra55 microscope. The system is equipped with a parabolic mirror for light collection and with both a photomultiplier and a charge-coupled device for detection.

\section{Results and discussion}

\subsection{Top-down fabrication of ordered arrays of GaN nanowires}

Ordered arrays of GaN NWs with different diameters and spacings in between NWs are produced simultaneously in an individual sample using a single sublimation step. Fig. 1 shows scanning electron micrographs of an exemplary NW array produced by annealing a patterned substrate at $825{ }^{\circ} \mathrm{C}$ for $20 \mathrm{~min}$. The $\mathrm{Si}_{x} \mathrm{~N}_{y}$ patches used as a mask for the sublimation process can be distinguished at the top of the GaN NWs by their darker contrast. The nominal NW diameter and spacing (distance between adjacent patches) values for this particular array are 90 and $100 \mathrm{~nm}$, respectively. The final average NW diameter, which roughly corresponds to the actual size of the $\mathrm{Si}_{x} \mathrm{~N}_{y}$ patches, is about $80 \mathrm{~nm}$. As can be observed in Fig. 1(a), the NW array is homogeneous on a large scale. Inside the patterned region, the NWs have rather vertical sidewalls [see Fig. 1(b)]. The NW side facets are, however, not as flat and well defined as in the case of self-assembled GaN NWs produced by conventional bottom-up growth approaches.,26-31 Specifically, 

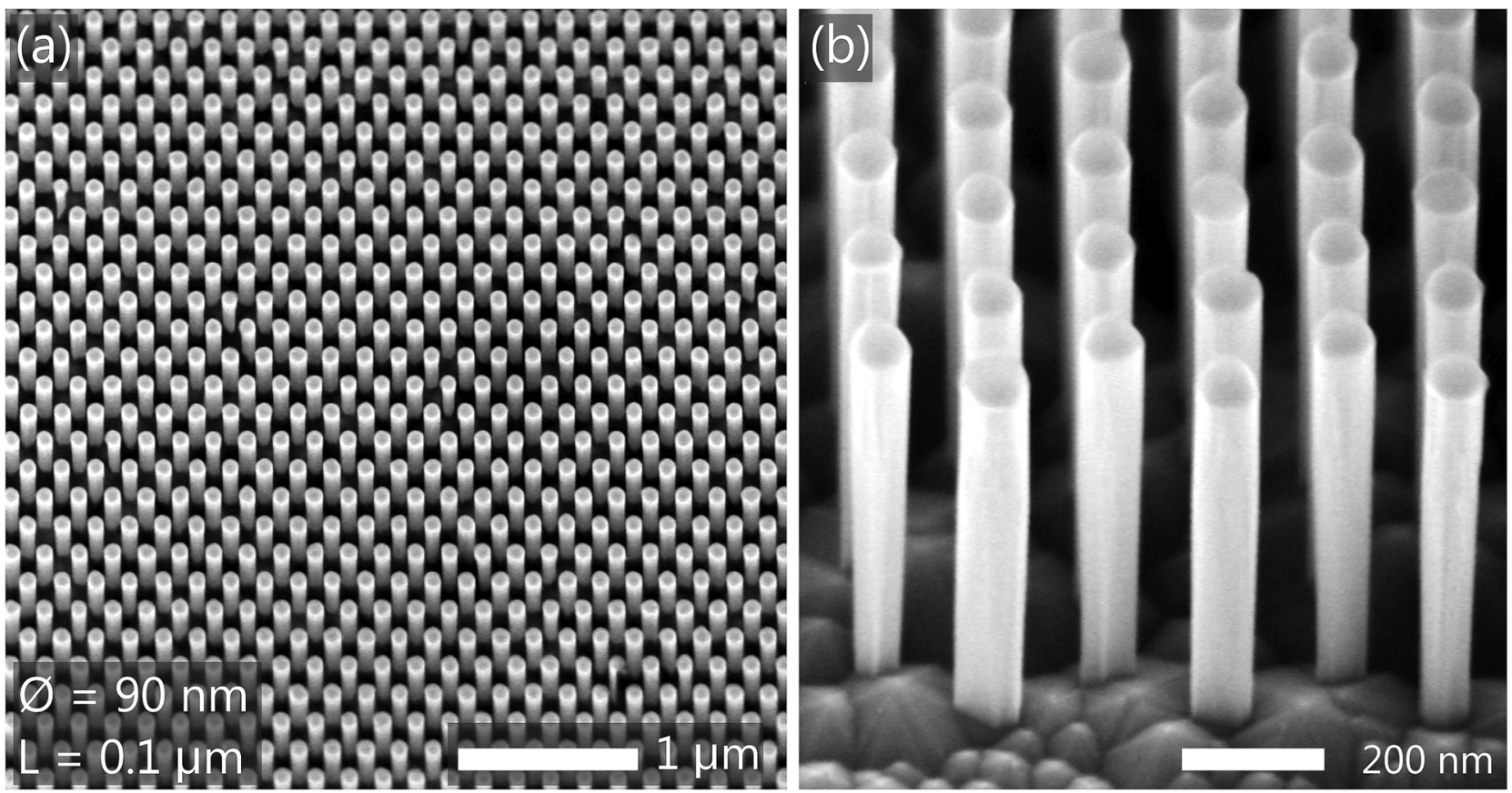

Fig. 1 ( $a$ and b) Bird's eye-view scanning electron micrographs with different magnifications of a GaN NW array produced by selective area sublimation. The nominal values of the NW diameter $(\varnothing)$ and the spacing $(L)$ are $90 \mathrm{~nm}$ and $0.1 \mu \mathrm{m}$, respectively. The micrograph shown in (b) is acquired at one of the edges of the patterned region.

we do not observe pronounced $M$-plane sidewall facets. The NW sidewalls are instead rather roundish, most likely because the $\mathrm{Si}_{x} \mathrm{~N}_{y}$ patches do not have a hexagonal but a circular shape (a representative scanning electron micrograph of the $\mathrm{Si}_{x} \mathrm{~N}_{y}$ patches before the thermal sublimation process is provided as ESI†).

Fig. 2 presents scanning electron micrographs of four additional NW arrays produced at the same time as the one shown in Fig. 1. These arrays differ from the previous one in either the NW diameter or the spacing. In all cases we observe the formation of homogeneous NW arrays with a very high yield. The yield only decreases due to the presence of TDs. The latter are easily recognized after the sublimation process because of the formation of hexagonal pits which are randomly distributed with a density of about $0.5 \times 10^{8} \mathrm{~cm}^{-2}$, lower than the nominal TD density of the parent GaN layer $\left(2 \times 10^{8} \mathrm{~cm}^{-2}\right)$. This discrepancy originates likely from the fact that the sublimation process, analogously to chemical etching, ${ }^{32}$ preferentially etches screw TDs. Note that according to the nominal TD density of the GaN layer, the average number of TDs per NW (estimated as the product of the TD density of the original GaN layer and the NW cross-sectional area ${ }^{15}$ ) amounts to 0.004 to 0.012 when the NW diameter is varied from 50 to $90 \mathrm{~nm}$. Consequently, even for the array with the larger NW diameter, approximately $99 \%$ of the NWs are expected to be free of TDs. ${ }^{18}$ The results shown in Fig. 1 and 2 demonstrate that homogeneous arrays of GaN NWs with various diameters and spacings can be obtained with identical sublimation parameters.

As can be seen in Fig. 2(c) and (d), the morphology of the initially flat GaN layer in between the $\mathrm{Si}_{x} \mathrm{~N}_{y}$ patches is clearly altered during the sublimation process. Fig. 3(a) presents a highly magnified scanning electron micrograph of an unpatterned GaN(0001) layer decomposed for $20 \mathrm{~min}$ at $825^{\circ} \mathrm{C}$. This micrograph reveals that the original (0001) surface gives way to the formation of three-dimensional islands with a six-fold symmetry and well-defined semi-polar facets. This result is in apparent contrast to the step-edge and layer-by-layer decomposition mechanisms reported in ref. 33 and 34, respectively. However, in these previous studies, where the decomposition process was analyzed at different temperatures, the GaN layer was not continuously decomposed (as in the present case), but a smooth (0001) surface was recovered prior to each (comparatively brief) decomposition step by depositing a thin GaN layer under conditions favoring step-flow growth. As a matter of fact, in ref. 33 it is also noted that continuous decomposition can result in surface faceting, a phenomenon that was tentatively ascribed to an enhanced sublimation rate near defects or/and grain boundaries. In the present study, the faceting under continuous decomposition is also detected in situ by RHEED. Fig. 3(b) and (c) present the RHEED patterns recorded along the

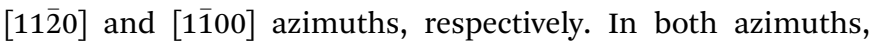
a transmission pattern is observed, accompanied by pronounced chevrons. These features, caused by the refraction and transmission diffraction of electrons entering and exiting crystal facets, can be used to derive the shape of the threedimensional objects from which they originate. ${ }^{35-40}$ As discussed in detail in the ESI, $\uparrow$ the analysis of the vertex angles allows us to conclusively conclude that, in agreement with the results reported by Damilano et al. in ref. 17, the facets of the islands seen in Fig. $3(\mathrm{a})$ are formed by $\{1 \overline{1} 03\}$ planes. 

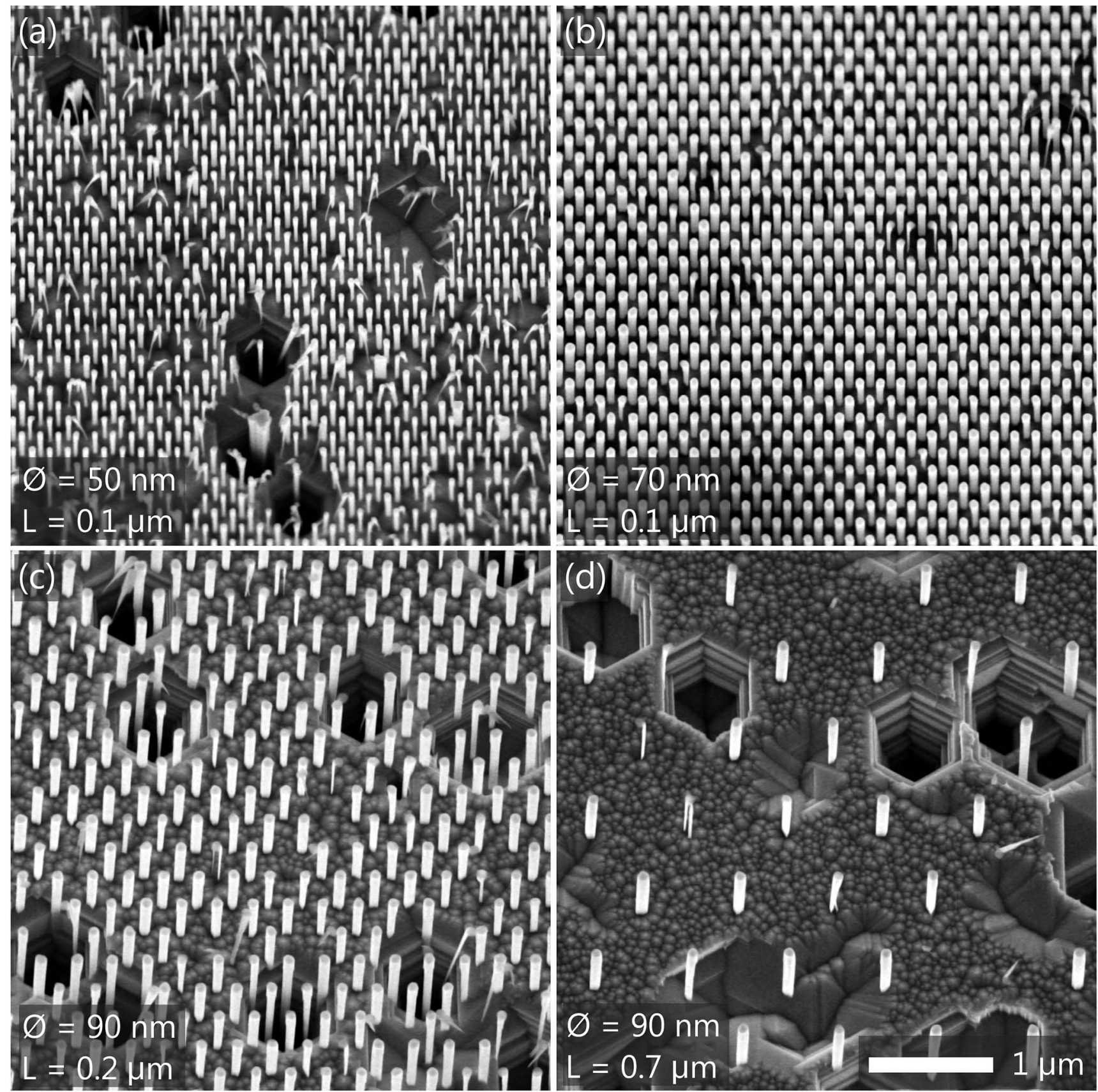

Fig. 2 (a-d) Bird's eye view scanning electron micrographs of NW arrays produced by selective area sublimation in the same run with varying nominal NW diameter $(\varnothing)$ and spacing $(L)$ values, as indicated in their corresponding labels. The magnification is the same for all micrographs and the scale bar is indicated in (d).

3.2 Thermal decomposition of GaN $\{1 \overline{10} 03\}$ facets: temperature dependence

To properly control the fabrication of nanostructures by SAS, it is essential to know the precise temperature dependence of the GaN decomposition rate in vacuum. The decomposition of $\mathrm{GaN}(0001)$ layers has been measured as a function of the temperature by different groups..$^{33,34,41-44}$ However, as discussed in Section 3.1, the (0001) surface is unstable against the formation of $\{1 \overline{1} 03\}$ facets, which are expected to decompose with a different rate. Hence, we next analyze the temperature dependence of the decomposition rate of $\{1 \overline{1} 03\}$ facets by measuring in situ the desorbing Ga flux at different substrate temperatures.

Fig. 4(a) shows the desorbing Ga flux as measured by QMS during the congruent thermal decomposition of an unpatterned $2^{\prime \prime}$ GaN wafer in vacuum. As shown in the graph, the substrate temperature $T$ is first increased up to $870{ }^{\circ} \mathrm{C}$ with a rate of $20 \mathrm{~K} \mathrm{~min}^{-1}$. Afterwards, the temperature is decreased in steps down to $786{ }^{\circ} \mathrm{C}$. To assess the desorbing Ga flux under-steady state conditions, we wait for the stabilization of 


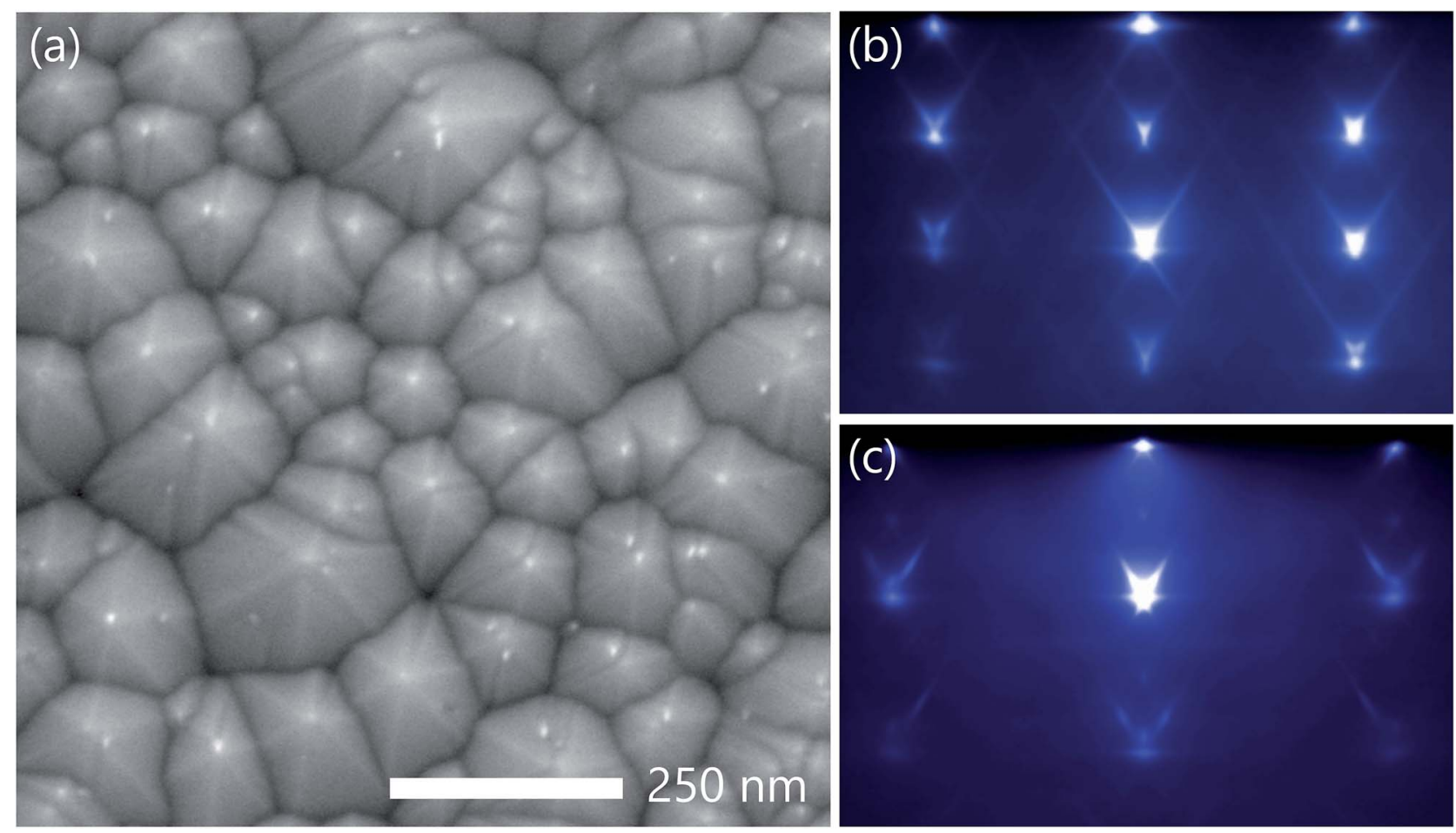

Fig. 3 (a) Bird's eye view scanning electron micrograph of a GaN layer after 20 min of annealing in vacuum at $825^{\circ} \mathrm{C}$. RHEED patterns acquired along the (b) [1120] and (c) [11̄00] azimuths after the thermal decomposition process.
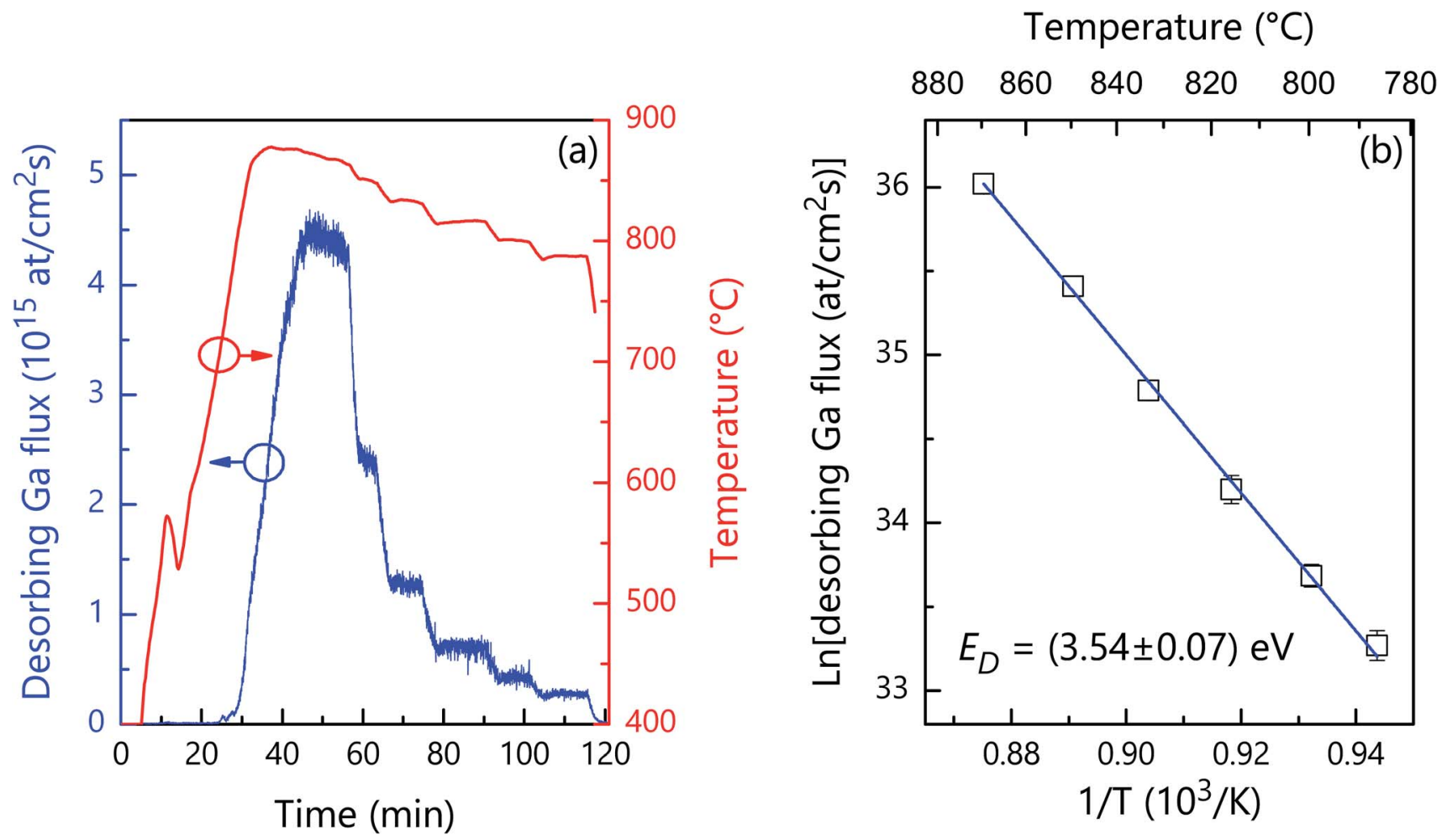

Fig. 4 (a) Change of substrate temperature and the resulting temporal evolution of the desorbing Ga flux (red and blue lines, respectively) during the decomposition of a GaN layer. (b) Arrhenius representation of the temperature dependence of the desorbing Ga flux during the decomposition of a $\{1 \overline{1} 03\}$-faceted GaN layer. The line shows the best fit that yields an activation energy of $E_{\mathrm{D}}=(3.54 \pm 0.07) \mathrm{eV}$. 
the substrate temperature after every temperature step. The measurements evidence that the desorbing Ga flux steadily decreases from $4.4 \times 10^{15} \mathrm{~cm}^{-2} \mathrm{~s}^{-1}\left(\approx 60 \mathrm{~nm} \mathrm{~min}^{-1}\right)$ at $870{ }^{\circ} \mathrm{C}$ down to $0.3 \times 10^{15} \mathrm{~cm}^{-2} \mathrm{~s}^{-1}\left(\approx 4 \mathrm{~nm} \mathrm{~min}^{-1}\right)$ at $786{ }^{\circ} \mathrm{C}$. The Arrhenius plot of the steady-steady state values of the decomposition rate of $\{1 \overline{1} 03\}$ facets $\left(\Phi_{\mathrm{D}}\right)$ is presented in Fig. 4(b). A fit of the data by

$$
\Phi_{\mathrm{D}}=A \exp \left(-E_{\mathrm{D}} / k_{\mathrm{B}} T\right)
$$

with the Boltzmann constant $k_{\mathrm{B}}$ yields a prefactor $A$ of $1.8 \times 10^{31}$ atoms per $\mathrm{cm}^{2}$ per $\mathrm{s}$ and an activation energy $E_{\mathrm{D}}$ of $(3.54 \pm$ $0.07) \mathrm{eV}$. When taking into account the increased surface area associated to the faceting, we find that the actual value of the exponential prefactor describing the thermal decomposition rate of $\{1 \overline{1} 03\}$ facets is reduced by a factor $\left(\tan (\theta)^{2}+1\right)^{1 / 2}$, where $\theta$ is the angle between the normal vectors of the $\{1 \overline{1} 03\}$ and $(0001)$ planes $\left(32.0^{\circ}\right)$. The actual value of the exponential prefactor is thus $1.58 \times 10^{31}$ atoms per $\mathrm{cm}^{2}$ per s. The corresponding values of $E_{\mathrm{D}}$ and $A$ measured by QMS in the case of a $\mathrm{GaN}(0001)$ plane are $(3.1 \pm 0.1) \mathrm{eV}$ and $1.17 \times 10^{29}$ atoms per $\mathrm{cm}^{2}$ per s. ${ }^{34}$ Consequently, even though the energy barrier for thermal decomposition is slightly higher in the case of the $\{1 \overline{1} 03\}$ facets, in the temperature range of interest, these facets decompose faster than the (0001) one due to the much higher exponential prefactor. These results are consistent with the idea that, while during growth the morphology of the crystal is governed by slow growing facets, during dissolution/thermal decomposition the crystal shape is dominated by fast desolving/decomposing crystal facets. ${ }^{45,46}$

\subsection{Luminescence from ordered arrays of GaN nanowires produced by selective area sublimation}

The luminescence from ordered arrays of GaN NWs is investigated by CL spectroscopy at $14 \mathrm{~K}$. Fig. 5(a) presents the near band-edge CL spectrum of the NW array shown in Fig. 1. For comparison, we have also included a second CL spectrum recorded on an unpatterned region of the same sample. Both spectra are dominated by a high-energy line originating from the radiative decay of free excitons (FX), and its first- and second-order longitudinal-optical phonon replica (LO) at lower energies. The dominance of free over bound exciton recombination in this specific experiment is due to both the comparatively high excitation density (on the order of several $10^{17} \mathrm{~cm}^{-3}$ ) and the resulting high effective carrier temperature [amounting to about $60 \mathrm{~K}$ for the spectra in Fig. 5(a), as determined by a fit to the high-energy slope of the free exciton line]. Two observations are worth to be stressed. First, the luminous intensity measured from the NW array is notably higher than that from the adjacent parent layer. This observation shows, most importantly, that the sublimation process does not degrade the internal quantum efficiency of the structure created, and furthermore, that the extraction efficiency of light is enhanced significantly over that of the planar reference. ${ }^{7,8}$ Second, the spectrum of the NW array is rigidly red-shifted by $3-4 \mathrm{meV}$ in comparison to the planar reference, as shown in the inset of Fig. 5(a) for the FX transition. This redshift results from the elastic relaxation of the residual compressive strain in the parent $\mathrm{GaN}(0001)$ layer grown on $\mathrm{Al}_{2} \mathrm{O}_{3}$. The same phenomenon, benefiting from the large surface-to-volume ratio of these nanostructures, was observed
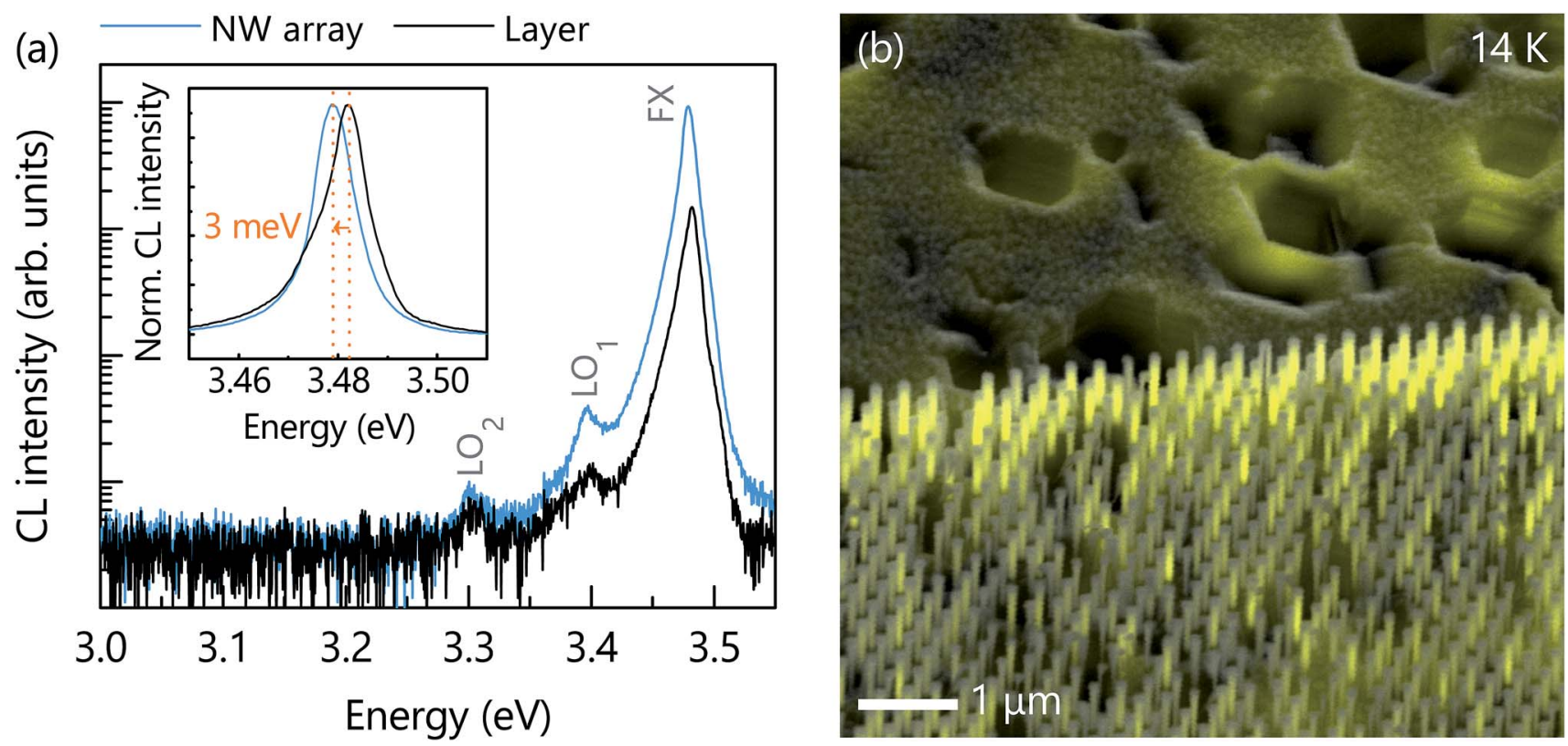

Fig. 5 (a) Near band-edge CL spectrum recorded at $14 \mathrm{~K}$ in bird's eye view geometry of the GaN NW array with a nominal NW diameter of 90 nm and a spacing of $0.1 \mu \mathrm{m}$. For comparison, the CL spectrum from the GaN layer surrounding the NW array (acquired under identical conditions) is also included. The emission from free excitons and its corresponding first and second order phonon replicas are labeled as $\mathrm{FX} \mathrm{LO}_{1}$, and $\mathrm{LO}_{2}$, respectively. The inset presents the normalized $\mathrm{CL}$ spectra of the NWs and the surrounding layer on a linear scale. (b) Superposition of a panchromatic $\mathrm{CL}$ intensity image recorded at $14 \mathrm{~K}$ (yellow) with the corresponding bird's eye view scanning electron micrograph for the same NW array as in (a). 
by different groups for pure GaN NWs as well as in the case of compressively strained (In, Ga)N quantum wells embedded into GaN NWs. ${ }^{14,17,19,47,48}$

The CL spectra shown in Fig. 5(a) were acquired immediately after exposing the sample to the electron beam. After prolonged exposure, the CL intensity in the patterned areas invariably quenches strongly. As an example, Fig. 5(b) shows a panchromatic CL map superimposed with its corresponding bird's eye view scanning electron micrograph. The CL from the patterned region appears to be inhomogeneous with several NWs being apparently not emitting at all. However, this quenching of the CL intensity upon irradiation is solely the result of carbonaceous depositions that introduce nonradiative recombination channels at the NW sidewalls, as discussed in detail in ref. 49.

\section{Summary and conclusions}

We have demonstrated the fabrication of ordered arrays of GaN NWs by SAS of pre-patterned GaN(0001) layers grown on $\mathrm{Al}_{2} \mathrm{O}_{3}$. In a single sample, we simultaneously produced different arrays with NW diameters and spacings ranging from 50 to $90 \mathrm{~nm}$ and 0.1 to $0.7 \mu \mathrm{m}$, respectively. The resulting NW sidewalls are vertical, but do not exhibit well-defined $M$-plane facets. The roundish shape of the NWs is attributed to the use of $\operatorname{Si}_{x} \mathrm{~N}_{y}$ patches with a circular shape as a mask for SAS. According to ref. 17, we expect the formation of $M$-plane sidewall facets when employing patches with a hexagonal shape properly oriented with respect to the GaN template underneath. During the sublimation process, the (0001) surface vanishes giving way to the formation of fast decomposing semi-polar $\{1 \overline{1} 03\}$ facets. The stability of these facets determines the thermal etching rate. We found that the $\{1 \overline{1} 03\}$ facets decompose following an Arrhenius-like temperature dependence with an activation energy of $(3.54 \pm 0.07) \mathrm{eV}$ and an exponential prefactor of $1.58 \times 10^{31}$ atoms per $\mathrm{cm}^{2}$ per s. Low-temperature CL experiments reveal a higher luminous intensity from the NW array thanks to an improved light extraction efficiency. The emission is red-shifted with respect to the one of the GaN layer because the large NW aspect ratio facilitates the elastic relaxation of residual strain. Although our CL experiments indicate that the sublimation process does not generate nonradiative recombination centers at the NW sidewalls, more conclusive results in this respect could be obtained by time-resolved photoluminescence spectroscopy. Such an analysis would be possible for GaN NWs containing (In,Ga)N quantum wells, for which the luminescence signals from the NWs and the GaN layer underneath would not spectrally overlap.

Selective area sublimation is, therefore, a suitable top-down approach to produce ordered arrays of GaN NWs with high luminous efficiency without the need of any elaborate chemical treatment. This fabrication method could be readily extended to other types of micro- and nanostructures as well as to additional material systems provided that they decompose congruently (to avoid the accumulation of constituent elements on the sample surface) and exhibit a marked anisotropy in the stability of its crystallographic planes. A prominent candidate for such experiments is $\mathrm{ZnO}$, another wide-bandgap semiconductor of interest for optical applications that shares many properties with GaN.

\section{Conflicts of interest}

There are no conflicts to declare.

\section{Acknowledgements}

We thank Katrin Morgenroth for her support during the preparation and characterization of the samples as well as for her dedicated maintenance of the molecular beam epitaxy system together with Carsten Stemmler and Hans-Peter Schönherr, Sebastian Meister and Sander Rauwerdink for patterning the substrates, and Anne-Kathrin Bluhm for her help during the acquisition of scanning electron micrographs. We are indebted to David van Treeck for numerous discussions on SAS, and to Vladimir Kaganer for discussions on the probability to find dislocations in NWs. Special thanks are due to Lutz Geelhaar for his continuous encouragement and support and a critical reading of the manuscript. Funding from the Bundesministerium für Bildung und Forschung through project FKZ:13N13662 is gratefully acknowledged. Sergio FernándezGarrido acknowledges the partial financial support received through the Spanish program Ramón y Cajal (co-financed by the European Social Fund) under grant RYC-2016-19509 from Ministerio de Ciencia, Innovación y Universidades.

\section{Notes and references}

1 M. Yoshizawa, A. Kikuchi, M. Mori, N. Fujita and K. Kishino, Jpn. J. Appl. Phys., 1997, 36, L459.

2 M. Sánchez-García, E. Calleja, E. Monroy, F. Sánchez, F. Calle, E. Muñoz and R. Beresford, J. Cryst. Growth, 1998, 183, 23.

3 S. Li and A. Waag, J. Appl. Phys., 2012, 111, 071101.

4 D. Zubia and S. D. Hersee, J. Appl. Phys., 1999, 85, 6492.

5 S. D. Hersee, A. K. Rishinaramangalam, M. N. Fairchild, L. Zhang and P. Varangis, J. Mater. Res., 2011, 26, 2293.

6 Q. Yue, K. Li, F. Kong, J. Zhao and W. Li, IEEE J. Quantum Electron., 2013, 49, 697.

7 N. P. Reddy, S. Naureen, S. Mokkapati, K. Vora, N. Shahid, F. Karouta, H. H. Tan and J. Chennupati, Nanotechnology, 2016, 27, 065304.

8 C. Hauswald, I. Giuntoni, T. Flissikowski, T. Gotschke, H. T. Grahn, L. Geelhaar and O. Brandt, ACS Photonics, 2017, 4, 52.

9 S. Nakagawa, T. Tabata, Y. Honda, M. Yamaguchi and H. Amano, Jpn. J. Appl. Phys., 2013, 52, 08JE07.

10 S. Kimura, H. Yoshida, K. Uesugi, T. Ito, A. Okada, S. Nunoue, S. Kimura, H. Yoshida, K. Uesugi, T. Ito and A. Okada, J. Appl. Phys., 2016, 120, 113104.

11 M. S. Mohajerani, M. Müller, J. Hartmann, H. Zhou, H.-H. Wehmann, P. Veit, F. Bertram, J. Christen and A. Waag, Jpn. J. Appl. Phys., 2016, 55, 05FJ09. 
12 S. Albert, A. Bengoechea-Encabo, J. Ledig, T. Schimpke, M. A. Sánchez-García, M. Strassburg, A. Waag and E. Calleja, Cryst. Growth Des., 2015, 15, 3661.

13 M. Müller, P. Veit, F. F. Krause, T. Schimpke, S. Metzner, F. Bertram, T. Mehrtens, K. Müller-Caspary, A. Avramescu, M. Strassburg, A. Rosenauer and J. Christen, Nano Lett., 2016, 16, 5340.

14 C. H. Chiu, T. C. Lu, H. W. Huang, C. F. Lai, C. C. Kao, J. T. Chu, C. C. Yu, H. C. Kuo, S. C. Wang, C. F. Lin and T. H. Hsueh, Nanotechnology, 2007, 18, 445201.

15 Q. Li, K. R. Westlake, M. H. Crawford, S. R. Lee, D. D. Koleske, J. J. Figiel, K. C. Cross, S. Fathololoumi, Z. Mi and G. T. Wang, Opt. Commun., 2011, 19, 25528.

16 Q. Li, J. B. Wright, W. W. Chow, T. S. Luk, I. Brener, L. F. Lester and G. T. Wang, Opt. Express, 2012, 20, 17873.

17 B. Damilano, S. Vézian, J. Brault, B. Alloing and J. Massies, Nano Lett., 2016, 16, 1863.

18 G. T. Wang, Q. Li, J. J. Wierer, D. D. Koleske and J. J. Figiel, Phys. Status Solidi A, 2014, 211, 748.

19 B. Damilano, V. S. Vézian, M. Portail, B. Alloing, J. Brault, A. Courville, B. V. Brändli, M. Leroux and J. Massies, J. Cryst. Growth, 2017, 477, 262.

20 L. Brockway, C. Pendyala, J. Jasinski, M. K. Sunkara and S. Vaddiraju, Cryst. Growth Des., 2011, 11, 4559.

21 B. Loitsch, D. Rudolph, S. Morkötter, M. Döblinger, G. Grimaldi, L. Hanschke, S. Matich, E. Parzinger, U. Wurstbauer, G. Abstreiter, J. J. Finley and G. Koblmüller, Adv. Mater., 2015, 27, 2195.

22 J. K. Zettler, P. Corfdir, C. Hauswald, E. Luna, U. Jahn, T. Flissikowski, E. Schmidt, C. Ronning, A. Trampert, L. Geelhaar, H. T. Grahn, O. Brandt and S. FernándezGarrido, Nano Lett., 2016, 16, 973.

23 J. Zúñiga-Pérez, V. Consonni, L. Lymperakis, X. Kong,

A. Trampert, S. Fernández-Garrido, O. Brandt, H. Renevier,

S. Keller, K. Hestroffer, M. R. Wagner, J. S. Reparaz, F. Akyol, S. Rajan, S. Rennesson, T. Palacios and G. Feuillet, Appl. Phys. Rev., 2016, 3, 041303.

24 N. Newman, J. Cryst. Growth, 1997, 178, 102-112.

25 S. Fernández-Garrido, J. K. Zettler, L. Geelhaar and O. Brandt, Nano Lett., 2015, 15, 1930.

26 A. Trampert, J. Ristić, U. Jahn, E. Calleja and K. H. Ploog, Proceedings of the 13th International Conference on Microscopy of Semiconducting Materials, IOP Conf. Ser. No., 2003, vol. 180, p. 167.

27 L. Largeau, D. L. Dheeraj, M. Tchernycheva, G. E. Cirlin and J. C. Harmand, Nanotechnology, 2008, 19, 155704.

28 W. Bergbauer, M. Strassburg, C. Kölper, N. Linder, C. Roder, J. Lähnemann, A. Trampert, S. Fündling, S. F. Li, H.-H. Wehmann and A. Waag, Nanotechnology, 2010, 21, 305201.
29 K. A. Bertness, S. Member, N. A. Sanford and A. V. Davydov, IEEE J. Sel. Top. Quantum Electron., 2011, 17, 847.

30 L. Geelhaar, C. Chèze, B. Jenichen, O. Brandt, C. Pfüller, S. Münch, R. Rothemund, S. Reitzenstein, A. Forchel, T. Kehagias, P. Komninou, G. P. Dimitrakopulos, T. Karakostas, L. Lari, P. R. Chalker, H. G. Mhairi and H. Riechert, IEEE J. Sel. Top. Quantum Electron., 2011, 17, 878.

31 O. Brandt, S. Fernández-Garrido, J. K. Zettler, E. Luna, U. Jahn, C. Chèze and V. M. Kaganer, Cryst. Growth Des., 2014, 14, 2246.

32 J. Weyher, S. Lazar, L. Macht, Z. Liliental-Weber, R. Molnar, S. Müller, V. Sivel, G. Nowak and I. Grzegory, J. Cryst. Growth, 2007, 305, 384.

33 N. Grandjean, J. Massies, F. Semond, S. Y. Karpov and R. A. Talalaev, Appl. Phys. Lett., 1999, 74, 1854.

34 S. Fernández-Garrido, G. Koblmüller, E. Calleja and J. S. Speck, J. Appl. Phys., 2008, 104, 033541.

35 T. Hanada, B.-H. Koo, H. Totsuka and T. Yao, Phys. Rev. B: Condens. Matter Mater. Phys., 2001, 64, 165307.

36 H. R. Gutiérrez, M. A. Cotta and M. M. G. de Carvalho, Appl. Phys. Lett., 2001, 79, 3854.

37 D. Pashley, J. Neave and B. Joyce, Surf. Sci., 2001, 476, 35.

38 C. Gaire, F. Tang and G.-C. Wang, Thin Solid Films, 2009, 517, 4509.

39 J. Lee, D. Schuh, M. Bichler and G. Abstreiter, Appl. Surf. Sci., 2004, 228, 306.

40 I. Ayahiko and P. I. Cohen, Reflection High-Energy Electron Diffraction, Cambridge University Press, 2004.

41 R. Groh, L. Bartha and J. I. Yankove, Phys. Status Solidi A, 1974, 26, 353.

42 R. Held, D. E. Crawford, A. M. Johnston, A. M. Dabiran and P. I. Cohen, Surf. Rev. Lett., 1998, 5, 913.

43 O. Ambacher, J. Vac. Sci. Technol., A, 1996, 14, 3532.

44 H. W. Choi, M. A. Rana, S. J. Chua, T. Osipowicz and J. S. Pan, Semicond. Sci. Technol., 2002, 17, 1223.

45 R. C. Snyder and M. F. Doherty, Materials, Interfaces and Electrochemical Phenomena, 2007, 53, 1337-1348.

46 M. R. Singh, N. Nere, H.-H. Tung, S. Mukherjee, S. Bordawekar and D. Ramkrishna, Cryst. Growth Des., 2014, 14, 5647.

47 M.-Y. Hsieh, C.-Y. Wang, L.-Y. Chen, T.-P. Lin, M.-Y. Ke, Y.-W. Cheng, Y.-C. Yu, C. P. Chen, D.-M. Yeh, C.-F. Lu, C.-F. Huang, C. C. Yang and J. J. Huang, IEEE Electron Device Lett., 2008, 29, 658.

48 V. Ramesh, A. Kikuchi, K. Kishino, M. Funato, Y. Kawakami, V. Ramesh, A. Kikuchi, K. Kishino, M. Funato and Y. Kawakami, J. Appl. Phys., 2010, 107, 114303.

49 J. Lähnemann, T. Flissikowski, M. Wölz, L. Geelhaar, H. T. Grahn, O. Brandt and U. Jahn, Nanotechnology, 2016, 27, 455706. 